See Analyst, Vol. III., No. 2 (1876), p. 49, for the sides of the 80th triangle; also Mathematical Visitor, Vol I., No. 3 (1879) p. 56 , and No. 5 (1880), p. 122, for sides of the 100 th triangle.

\title{
Artemas Martin.
}

Formula for Centrifugal Force.-The object of this note is to suggest finding the formula for centrifugal force as an exercise on space-rate of change of energy.

Let the uniform circular motion be that of a particle of mass $m$ travelling with speed $v$ in a circle centre $O$ and radius $r$, and let the motion be regarded as resolved into two linear motions with reference to rectangular axes $\mathrm{X}^{\prime} \mathrm{OX}, \mathrm{Y}^{\prime} \mathrm{OY}$. Let $\mathrm{MP}$ be the ordinate of any point $P$ on the circle.

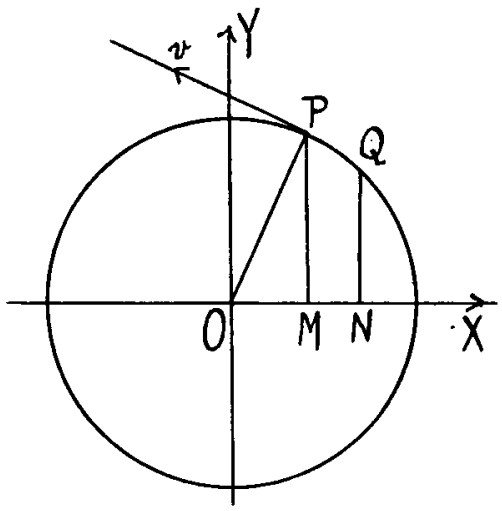

Then

$x$-component of velocity at $\mathrm{P}=v \sin \mathrm{XOP}=\frac{v \cdot \mathrm{MP}}{r}$.

Therefore

kinetic energy of $x$-linear motion at $\mathrm{P}=\frac{1}{2} m, \frac{v^{2} \cdot \mathrm{MP}^{2}}{r^{2}}$

Similarly, if $Q$ be a point on the circle near to $P$,

kinetic energy of $x$-linear motion at $\mathrm{Q}=\frac{1}{2} \frac{m v^{2}}{r^{2}} . N Q^{2}$. 
Therefore

$$
\begin{aligned}
\text { change of energy } & =\frac{1}{2} \cdot \frac{m v^{2}}{r^{2}}\left(\mathrm{MP}^{2}-\mathrm{NQ}^{2}\right) \\
& =\frac{1}{2} \cdot \frac{m v^{2}}{r^{2}}\left(\mathrm{ON}^{2}-\mathrm{OM}^{2}\right) \\
& =\frac{1}{2} \cdot \frac{m v^{2}}{r^{2}} \cdot \mathrm{MN}(\mathrm{ON}+\mathrm{OM}) .
\end{aligned}
$$

Therefore

average force in the interval $=$ change of energy $\div \mathrm{MN}$

$$
=\frac{1}{2} \cdot \frac{m v^{2}}{r^{2}}(\mathrm{ON}+\mathrm{OM}) \text {. }
$$

Therefore in the limit,

$$
x \text {-component of force at } \mathrm{P}=\frac{m v^{2}}{r^{2}} . \mathrm{OM} \text {. }
$$

Similarly,

$$
y \text {-component of force at } \mathrm{P}=\frac{m v^{2}}{r^{2}} . \mathrm{MP} \text {. }
$$

Since these are proportional to $\mathrm{OM}$ and $M P$, the resultant force acts along the radius, and its value is

$$
\frac{m v^{2}}{r^{2}} \cdot \sqrt{\mathrm{OM}^{2}+\mathrm{MP}^{2}} \text { or } \frac{m v^{2}}{r} \text {. }
$$

Indeed it is obvious from the beginning that the force is always at right angles to the direction of motion; for the energy of the motion is constant.

W. ANderson.

Deduction of Propositions on Tangents from Propositions on Secants.-In Vol. XXII., p. 64, of the Proceedings of the Society, Professor Gibson called attention to difficulties in the treatment of tangents by the method of limits. The aim of the following remarks is to show how young students may become accustomed to regard a tangent as the limit of a secant, and at the same time to furnish a type of exercise which might encourage initiative and research in the early study of Geometry.

The relation of tangent to secant in the geometry of the circle has a well known illustration in the deduction of Euc. III., 32, from Euc. III., 21. If, in Fig. 1, DB be produced to $\mathrm{E}$, BE will 